\title{
Interventional pain management during SARS-CoV-2 pandemic
}

\author{
Savas Comlek ${ }^{1, *}$, Aylin Ordu ${ }^{2}$
}

${ }^{1}$ Gayrettepe Florence Nightingale Hospital, Department of Anesthesiology and Reanimation, Gayrettepe, 34349

Istanbul, Turkey

${ }^{2}$ Istanbul Florence Nightingale Hospital, Department of Molecular Microbiology Laboratory, Sisli, 34381 Istanbul, Turkey

\section{*Correspondence}

savatc@gmail.com

(Savas Comlek)

\begin{abstract}
Background: SARS-CoV-2 virus resulted in a sudden dreadful outbreak, posing a serious global public health problem consuming most of the healthcare resources. Requirement to allocate the existing resources for SARS-CoV-2 management during the pandemic has led to significant challenges in pain management as well as in other healthcare services.

Objectives: This study aimed to report the outcomes of an interventional pain management unit of a COVID-free hospital, where a fraction of medical services is being provided through telemedicine, and where patients and healthcare workers are screened periodically, in addition to the implementation of guideline-recommended key precautions.
\end{abstract}

Study design: Retrospective chart review.

Setting: Pain clinic of a private COVID-free hospital.

Methods: A total of 83 patients asymptomatic for SARS-CoV-2 and scheduled for interventional pain management (epidural injection, epidural neuroplasty, peripheral nerve and sympathetic ganglion block, and radiofrequency interventions for nonmalignant pain; celiac-splanchnic impar ganglion neurolysis and epidural-spinal port catheter implantation for malignant pain) were screened using reverse transcriptase polymerase chain reaction (RT PCR), Immunoglobulin G and Immunoglobulin M antibodies, and computerized tomography (CT) imaging prior to the intervention and were then followed-up for a minimum duration of 30 days. All healthcare workers of the hospital as well as the staff in the pain management center were monitored with RTPCR tests performed every 5 days. The efficacy of pain management interventions was assessed using a 10-point visual analogue scale (VAS), while a 5-point post-intervention questionnaire was used to measure the patient satisfaction regarding telemedicine practices, where 5 indicates maximum satisfaction.

Results: Patients were finally diagnosed as follows: 2 (2.4\%) patients, PCR-confirmed SARS-CoV-2 infection; 3 (3.6\%) patients, previous SARS-CoV-2 infection; 13 (15.7\%) patients, suspected SARS-CoV-2 infection; and $65(78.3 \%)$ patients, no SARS-CoV2 infection. Three out of the 517 healthcare workers $(0.6 \%)$ had PCR positivity in routine screening performed throughout the study, and all these three subjects were asymptomatic. Following interventional treatment, VAS scores were significantly reduced at 2 weeks when compared to baseline $(3.9 \pm 1.3$ vs. $8.6 \pm 1.0, P<0.001)$, and this reduction was maintained until month 1 . Mean satisfaction scores for the communication with the physician using telemedicine methods and for the follow-up management using telemedicine methods were $3.0 \pm 0.9$ and $3.7 \pm 0.6$, respectively. None of the patients had steroid-related side effects and none developed new-onset SARS-Cov-2 confirmed or suspected infection throughout the study.

Limitations: Retrospective design and relatively small sample size.

Conclusion: Our findings suggest that effective diagnostic tests/imaging studies aimed at detecting infected patients and healthcare workers, and telemedicine practices contribute to the provision of safe and feasible pain management practices during the SARS-CoV-2 pandemic.

\section{Keywords}

SARS-CoV-2; COVID-19; Pandemic; Pain management; Interventional pain management 


\section{Introduction}

Coronavirus disease (COVID-19) is a very contagious infection caused by a new strain known as SARS-CoV-2 identified in humans; and the sudden dreadful outbreak of the COVID-19 by the end of 2019 spread around the world, posing a serious global concern for public health [1].

Globally, pain and pain-related conditions represent a major cause of disability and disease burden that needs adequate management [2]. Access to pain management services has been described as a fundamental human right owing to the critical role of chronic pain in the physical and psychosocial wellbeing of individuals $[3,4]$.

The current pandemic of SARS-CoV-2 has led to significant challenges in pain management, due to the requirement to allocate the existing resources to the most urgent healthcare needs in a most efficient way. As a result of a concerted effort between scientific authorities and policy makers to provide solutions for such problems, several guidelines have been developed to inform physicians on methods to maintain a good quality pain management service [5-7].

On one hand, COVID-free hospitals have been proposed for maintaining the functionality of health services, and on the other hand, due to relatively high prevalence of asymptomatic cases, screening and diagnostic tests such as PCR, antibody assays, and radiological imaging studies are now being used for patients scheduled for surgery or other interventions [8, 9]. The estimated rate of asymptomatic infections ranges between 18 and $81 \%$ [10]. Although the public health importance of routine screening in patients as well as in healthcare workers is well known, publications reporting on interventional pain management in COVID-free hospitals and on the outcomes and value of screening among patients and healthcare personnel have been relatively scarce.

In an effort to eliminate the role of hospitals as a source of infection, provision of healthcare through mobile phones and computers (i.e. telemedicine as traditionally defined) has been advocated as an effective means for pain management, particularly for patients with chronic pain [11]. However, the utility of telemedicine in interventions performed in pain management units and the resultant patient satisfaction levels have not been adequately studied.

This study aimed to examine the frequency of SARS-CoV2 infection among candidates for interventional pain management and risks of new-onset disease development in patients and healthcare workers, in an attempt to have an idea on the feasibility of such interventions in the pandemic setting. Thus, the study focuses on both healthy individuals and infected patients. Therefore, we decided to publish promising data obtained at an interventional pain management unit of a COVID-free hospital, where a fraction of medical services is being provided through telemedicine, and where patients and healthcare workers are screened periodically, in addition to the implementation of guideline-recommended key precautions such as triage, use of protective equipment, and arrangement of physical conditions.

\section{Patients and methods}

\subsection{Patients}

In this study involving a total of 83 participants undergoing interventions for pain management (epidural injection, epidural neuroplasty, peripheral nerve and sympathetic ganglion block, and radiofrequency interventions for nonmalignant pain; celiac-splanchnic impar ganglion neurolysis and epidural-spinal port catheter implantation for malignant pain) between $20^{\text {th }}$ March 2020 and $20^{\text {th }}$ June 2020, during which a marked increase was observed in the number of cases in Turkey, patients asymptomatic for SARS-CoV-2 were screened using RT PCR, IgG and IgM antibodies, and CT imaging prior to the intervention, and were then followed-up for a minimum duration of 30 days. Patient data obtained in accordance with the operational guidelines designed for the pandemic as well as demographic data were retrospectively evaluated. Table 1 shows demographical and clinical characteristics of the patients including indications and type of pain interventions. The study was approved by Demiroglu Bilim University Ethics Committee for Clinical Studies (number, 44140529/4218; date, June 23, 2020) and performed in accordance with the ethical standards as laid down in the 1964 Declaration of Helsinki and its later amendments.

TA B L E 1. Demographical and clinical characteristics of the patients.

\begin{tabular}{lc} 
Characteristics & $\mathbf{n}=\mathbf{8 3}$ \\
Age, y (mean \pm SD) & $55.3 \pm 14.0$ \\
Female sex & $51(61.4 \%)$ \\
Weight, $\mathrm{kg}($ mean $\pm \mathrm{SD})$ & $69.2 \pm 9.2$ \\
Malign disease & $28(33.7 \%)$ \\
Indication for intervention & \\
Malignancy related pain & $23(27.7 \%)$ \\
Non-malignancy related pain & $55(66.3 \%)$ \\
CTX port catheter implantation & $5(6.0 \%)$ \\
Steroid injection & $52(62.7 \%)$ \\
Systemic steroids & $6(7.2 \%)$ \\
\hline
\end{tabular}

Unless otherwise stated, data is presented as $n(\%)$. SD, standard deviation; CTX, chemotherapy.

\subsection{Screening and infection control measures}

\subsubsection{Initial evaluations}

Patients requiring interventional pain management in a pain center were initially evaluated through digital communication tools, either with video or telephone calls. During this interview, information on the primary condition, presence/absence of common SARS-CoV-2 related symptoms (high fever, cough, dyspnea, and fatigues, less common symptoms like aches and pains, sore throat, diarrhea, conjunctivitis, headache, loss of taste or smell, a rash on the skin, or discoloration of fingers or toes), as well as the history of suspected contact with infected subjects, was gathered. For 
those patients who were scheduled for an intervention, an RT-PCR assay (NAAT, nucleic acid amplification test) was performed 24 hours prior to the procedure in the biological safety units located outside the hospital building, in addition to IgG and IgM antibody testing in isolated blood sampling units that was performed on the day of the intervention.

\subsubsection{Procedures for patients with the infection}

The procedure was postponed for 15 days for those with active infection based on PCR or antibody testing. Furthermore, those with no signs of active infection underwent computed tomographic (CT) examination of the chest. Those with suspicious CT imaging, the intervention was not deferred, and those patients were admitted to isolated beds in the pain management unit and were discharged on the same day of intervention. Those with no suspicious CT findings were admitted to standard patient wards and were discharged on the same day.

\subsubsection{Follow-up}

RT-PCR, antibody testing, and CT imaging were periodically repeated based on the chemotherapy scheme and clinical condition in patients with cancer. In interventions for non-cancer patients, those with suspicious laboratory findings or clinical symptoms, or those who required a postponement of the procedure, the tests were repeated. A 5-day period was allowed for safety, and digital communication tools or telephone calls were preferred outside this period in addition to the use of electronicprescriptions negating the need for face-to-face patient communication. All healthcare workers of the hospital as well as the staff in the pain management center were monitored with RT-PCR tests performed every 5 days, and antibody testing and chest CT were performed if required.

\subsection{Assessments}

\subsubsection{PCR testing}

RT-PCR tests were performed in a Rotor- GeneQ-QIAGEN device (QIAGEN, Germany, manufactured in Malaysia) using bio-speedy SARS-CoV-2 (2019-ncov) qPCR detection kits (Bioeksen R\&D Technologies Limited Company, Turkey). Fingertip blood samples were used for point-of-care antibody testing (Orient Gene Biotech COVID-19 IgG/IgM Rapid Test Cassette; Zhejiang Orient Gene Biotech, Zhejiang, China; reference GCCOV-402a).

\subsubsection{Computerized tomography evaluation}

CT findings were assessed using COVID-19 Reporting and Data System (CO-RADS) classification [12]. In addition, risk groups based on American Society of Interventional Pain Physicians (ASIPP) and Centers for Disease Control (CDC) definitions were recorded.

\subsubsection{Assessment of the efficacy of pain interventions}

The efficacy of pain management interventions was assessed using a 10-point visual analogue scale (VAS), where 10 represents the worse pain, while post-intervention questionnaires were used to measure the patient satisfaction regarding telemedicine practices adopted for pain management during the pandemic. The satisfaction level was scored by the patients using a 5-point scale, where 5 indicates maximum satisfaction and 1 indicates minimum satisfaction.

\subsection{Statistical analysis}

SPSS (Statistical Package for Social Sciences) version 21 software (SPSS Inc., Chicago, IL) was used for data analysis. Results are presented as mean \pm standard deviation, median (range), or number (percentage), where appropriate. Hypothesis tests and graphical methods were used to test normality. Friedman test was used to examine the significance of changes in VAS scores over time (baseline, week 1, month 1). Post hoc within-subject comparisons of VAS scores were done by Wilcoxon signed-rank test with Bonferroni correction. Twosided $P$ values $<0.05$ were considered indication of statistical significance. For the purpose of Bonferroni correction, a $P$ value $<0.017$ was considered indication of statistical significance for pairwise comparisons between different time points.

\section{RESULTS}

\subsection{Patients}

Table 1 shows demographical and clinical characteristics of the patients. Majority had treatment for non-malignant pain (66.3\%) and only one-third had accompanying malignant condition. Almost two-thirds of the patients received local steroid injection (methylprednisolone acetate) during the procedure but none of them experienced steroid-related side effects. None developed new-onset SARS-CoV-2 confirmed or suspected infection throughout the study and none of the patients with suspected COVID-19 at baseline and received pain treatment had disease progression.

\subsection{SARS-CoV-2 related data}

According to ASIPP risk category, 70 (84.3\%), 12 (14.5\%), and $1(1.2 \%)$ patient had low, intermediate, and high risk, respectively. According to CDC risk category, 36 (43.4\%) and 47 (56.6\%) had intermediate and high risk, respectively. Patients were finally diagnosed as follows: $2(2.4 \%)$ patients, PCR-confirmed SARS-CoV-2 infection; 3 (3.6\%) patients, previous SARS-CoV-2 infection; 13 (15.7\%) patients, suspected SARS-CoV-2 infection; and $65(78.3 \%)$ patients, no SARS-CoV-2 infection. SARS-CoV-2 was ruled out in four patients initially categorized as suspected SARS-CoV-2 during more detailed investigation, thus categorized as no SARSCoV-2.

Two males at 43 and 44 years of age without malignancy was diagnosed with PCR-confirmed SARS-CoV-2 with positive IgM antibody and CO-RADS4 CT finding. Both had high CDC risk but intermediate ASIPP risk. PCR became negative and IgM disappeared in both at two weeks and only one developed IgG antibody at one month. After a delay of 15 days, both patients had epidural neuroplasty with steroid and hyaluronidase injection. Three patients had a history of previous SARS-CoV-2 injection with negative PCR on admission. Two of them had IgG and CO-RADS1 CT finding 
TA B L E 2. Summary of the patients diagnosed with suspected SARS-CoV-2 (PCR negative cases).

\begin{tabular}{|c|c|c|c|c|c|c|c|}
\hline \multicolumn{3}{|c|}{ No Age/Sex Malignancy } & \multirow{2}{*}{$\begin{array}{c}\text { ASIPP/CDC Risk } \\
\text { Low/High }\end{array}$} & \multicolumn{3}{|c|}{ Antibody CO-RADS Baseline/30-day Steroid injection } & \multirow[t]{2}{*}{ Systemic steroid } \\
\hline 1 & 49/M & - & & - & $3 / 3$ & Yes & \\
\hline 2 & $81 / \mathrm{F}$ & - & Low/High & - & $3 / 1$ & Yes & \\
\hline 3 & $61 / \mathrm{F}$ & - & Moderate/Moderate & - & $3 / \mathrm{NE}$ & Yes & \\
\hline 4 & $41 / \mathrm{F}$ & Yes & Low/High & - & $3 / \mathrm{NE}$ & & \\
\hline 5 & $64 / \mathrm{F}$ & Yes & Low/High & - & $3 / \mathrm{NE}$ & & \\
\hline 6 & $44 / \mathrm{M}$ & Yes & Moderate/High & - & $4 / 3$ & & \\
\hline 7 & $74 / \mathrm{F}$ & Yes & Low/High & - & $3 / 1$ & & \\
\hline 8 & $59 / \mathrm{F}$ & Yes & Moderate/High & - & $3 / 1$ & & \\
\hline 9 & $35 / \mathrm{F}$ & Yes & Low/High & - & $4 / 2$ & & \\
\hline 10 & $55 / \mathrm{M}$ & Yes & Low/High & - & $4 / 3$ & & Yes \\
\hline 11 & $42 / \mathrm{F}$ & Yes & Low/High & - & $4 / 4$ & & Yes \\
\hline 12 & $62 / \mathrm{F}$ & - & Low/Moderate & $\operatorname{IgM}^{*}$ & $3 / 3$ & Yes & \\
\hline 13 & 49/M & - & Low/High & $\mathrm{IgM} \dagger$ & $2 / 1$ & Yes & \\
\hline
\end{tabular}

*disappeared at two weeks, †disappeared at one month. ASIPP, American Society of Interventional Pain Physicians; CDC, Centers for Disease Control; CO-RADS, COVID-19 Reporting and Data System; M, male; F, female; NE, not evaluated

on admission, whereas the remaining patient had CO-RADS3 CT finding and did not develop IgG during follow-up. Table 2 summarizes the cases with suspected SARS-CoV-2. The procedure was delayed for 15 to 30 days in five patients including the two patients with PCR-confirmed SARS-CoV-2. No new SARS-CoV-2 cases were seen during follow-up.

Three out of the 517 healthcare workers $(0.6 \%)$ had PCR positivity on routine screening performed throughout the study, and all these three subjects were asymptomatic.

\subsection{VAS scores and satisfaction from telemedicine}

A significant difference was evident in VAS scores between the three time points (baseline, week 2, and month one) $(P<$ 0.001). Initially, VAS scores were significantly reduced at 2 weeks when compared to baseline $(3.9 \pm 1.3$ vs. $8.6 \pm 1.0, P$ $<0.001$ ), and this reduction was maintained until the end of month 1 without further reduction.

Satisfaction scores were available for 74 patients $(89.2 \%)$. Mean satisfaction score for the communication with the physician using telemedicine methods was $3.0 \pm 0.9$ (median, 3; range, 1-5). Mean satisfaction score for the follow-up management using telemedicine methods was $3.7 \pm 0.6$ (median, 4; range, 2-5).

\section{Discussion}

The novelty of this study is that it particularly examines the feasibility and safety of interventional pain management, which may of very utmost importance for the patient wellbeing, in such pandemic setting. In this study, it was possible to perform interventional pain management practices efficiently in a COVID-free hospital setting using an integrated approach.

PCR screening of asymptomatic populations prior to surgery/interventions has revealed a SARS-CoV-2 positivity rate of $0 \%$ to $13.7 \%$ [13-17]. These differences in reported rates of positivity have been attributed to the fact that "the incidence of subclinical infection may probably vary according to the prevalence rates in different communities [16].

While some studies showed that day surgery may be effectively managed with low risk of COVID infection using a number of precautionary measures such as PCR screening, usage of protective equipment, re-arrangement of physical conditions, and reducing the traffic in the surgery room and number of patient visitors [18], others underscored the value of IgM and IgG antibody testing in combination with PCR prior to elective surgical procedures [19].

In a study by EM Huybens et al. investigating the preferred screening method for COVID-19 among 386 asymptomatic patients undergoing surgery or interventional procedures, it was concluded that screening is required prior to such procedures [13]. These authors underscored the importance of a questionnaire gathering information on COVID-19 symptoms and recommended PCR testing in certain risk groups, while they also concluded that a thoracic CT examination with CO-RADS classification will not provide additional screening benefits in conditions of low COVID-19 prevalence. Similarly, the rate of infection declined following the completion of our study, and the routine thoracic $\mathrm{CT}$ screening before pain management interventions was discontinued.

Also, as was the case with our facility, it has been reported that most types of elective surgical procedures could be safely undertaken in COVID-free hospital conditions [20].

Healthcare workers comprise more than $10 \%$ of all SARSCoV-2 cases in Italy and Spain, and $6.5 \%$ of the cases in Turkey $[21,22]$. The rate of undiagnosed and asymptomatic cases has been reported to be $3.3 \%$ among healthcare workers [23]. Inadequate supply of protective equipment plays an important role in this process, and healthcare workers are not only exposed to physical risk of disease and death, but also to the risk of mental and psychological trauma. In one 
study by Lucy Rivett et al. involving 1032 asymptomatic healthcare workers, $3 \%$ of the subjects tested positive for SARS-CoV-2, and the authors emphasized the important role of asymptomatic transmission [24]. In the current study, the corresponding figures were much lower, and only 3 of the 517 healthcare workers $(0.6 \%)$ had PCR positivity on routine screening performed throughout the study, and all these three subjects were asymptomatic. These figures may be accounted for by the implementation of strict infection control measures, comprehensive screening of asymptomatic patients before the procedures, as well as the COVID-free status of our hospital.

The reported rates of mortality among SARS-CoV-2 infected patients undergoing minor and major surgery are $16.3 \%$ and $26.9 \%$ [25], indicating the importance of the detection of infected cases before such procedures. Although the definitive diagnosis of SARS-CoV-2 infection is established by RT-PCR positivity, some patients do not test positive despite clinical and radiological findings consistent with the disease, and these cases cannot be classified as having definitive SARS-CoV2. This has led to the definition of two different diagnostic categories, i.e. probable and definitive SARS-CoV-2, by the World Health Organization [26].

The common COVID imaging findings on CT examination (bilateral ground glass opacities) are not pathognomonic for SARS-CoV-2, and may also occur in other viral pneumonias (e.g. influenza, adenovirus) as well as in certain noninfectious conditions [21]. In our patient series, 4 of the 19 suspected cases based on thoracic CT findings were subsequently diagnosed with non-COVID viral infection based on bronchoalveolar lavage examination. Previous assessments of the diagnostic value of RT PCR and thoracic CT examinations suggested a high diagnostic sensitivity for the $\mathrm{CT}$ imaging. For example, in one previous study while only $59 \%$ of the patients had RT PCR positivity, this figure was $88 \%$ with thorax CT. Therefore, some authors suggested primary use of thorax CT for the detection of COVID-19 infected cases in epidemic areas [9]. In order to standardize thoracic CT findings, which are associated with high sensitivity but low specificity, CO-RADS classification has been proposed as a means for defining the level of suspicion in pulmonary involvement [12].

In addition to RT PCR or other antigen detection methods, antibody tests may also assist in determining the presence/absence of infection or in confirming the diagnosis (even if asymptomatic) among those with suspected acute infection [27].

Apart from interventional pain management methods, several measures may be helpful in preventing and alleviating pain during the pandemic. For example, including natural vegetables and fruits containing antioxidants into the diet of patients with chronic pain may help to reduce the reactive oxygen species responsible for potentiating the pain and inflammatory responses [28]. Such a strategy may also help to reduce heart attacks [29]. In addition, curfews and lockdowns implemented during COVID-19 pandemic have led to decreased physical activity, and because of such sedentary behavior the incidence of musculoskeletal pain is expected to rise [30, 31]. However, individuals can adopt simple self-therapeutic approaches with home-based exercises to alleviate pain and postural problems during the pandemic $[30,31]$.
Even before the SARS-CoV-2 pandemic, telemedicine and e-health methods had been introduced for use as a means for managing health services [32-35]. With regard to patient satisfaction with such methods, positive results were published [36]. Again, the benefits of telemedicine in pandemics or in other public health emergencies have been well documented $[11,37]$. Similarly, the advantages of telemedicine in protecting healthcare workers and patients alike during the SARSCoV-2 pandemic were also strongly highlighted, with associated cost-savings [38, 39]. In our study, initial patient interview and screening for SARS-CoV-2 symptoms as well as patient follow-up was managed through telemedicine practices, and e-prescriptions were delivered, leading to high patient satisfaction rates. This may also represent one contributing factor for the low rates of infection among our personnel.

One of the most controversial subjects in pain management during SARS-CoV-2 pandemic involves the use of opiates and steroids, particularly with respect to the effects on the immune system. The effect of opioids on the immune system is rather complicated, varying depending on the type of opioid, dosage, and type of immunity [40]. Although a relatively increased risk of infection with chronic opiate use has been suggested [41], the immunosuppressive effects of pain itself should also be considered [42].

Increased risk of infection, particularly regarding influenza virus, has been reported for steroid use [43], although most experts and health authorities hold the view that "the use of epidural steroid injections and other procedures may be continued". It has also been suggested that the lowest effective dose should be used, and patients should be informed regarding the risk of potential infection and immunosuppression [44]. Guidelines point out to a potentially higher risk among patients with active infection [44]. On the other hand, use of methylprednisolone was associated with positive effects on the disease course and improvement in lung functions, without compromising the formation of SARS-CoV-2-specific IgG formation [45]. WHO recommends the use of corticosteroids in SARS-CoV2 management in certain conditions [46].

The most important limitation of our study is the small sample size. Therefore, further studies with larger sample size are warranted to reach firmer conclusions. Another limitation is the retrospective design. Inclusion of other assessment tools such as Oswestry Disability Index (ODI), Western Ontario and McMaster Universities Osteoarthritis Index (WOMAC), and other Quality of Life indexes would be more informative on the benefit of the pain management in this pandemic era.

\section{Conclusions}

Our findings support that pain management interventions are feasible and relatively safe during the SARS-Cov-2 pandemic era. This may allow many interventions to be performed and contribute to the patient well-being, provided that effective diagnostic tests/imaging studies aimed at detecting infected patients and healthcare workers are utilized. In addition, telemedicine contributes to the provision of safe and feasible pain management through avoidance of direct contact and high patient satisfaction. 


\section{AUTHOR CONTRIBUTIONS}

Savas Comlek and Aylin Ordu fully contributed to the study conception and design, material preparation, data collection and analysis. Savas Comlek drafted the manuscript, Savas Comlek and Aylin Ordu developed the manuscript and critically reviewed and revised it. Finally, both authors read and approved the final version of the manuscript.

\section{ETHICS APPROVAL AND CONSENT TO PARTICIPATE}

The study was approved by Demiroglu Bilim University Ethics Committee for Clinical Studies (number, 44140529/4218; date, June 23,2020 ) and performed in accordance with the ethical standards as laid down in the 1964 Declaration of Helsinki and its later amendments.

\section{ACKNOWLEDGMENT}

The authors acknowledge the institutional support from Gayrettepe Florence Nightingale Hospital and Demiroglu Bilim University. They also thank numerous patients and healthcare workers who participated in this study. In addition, they would like to express their gratitude to all those who helped during the writing of this manuscript and they thank all the peer reviewers for their opinions and suggestions.

\section{FUNDING}

There was no external funding in the preparation of this manuscript.

\section{CONFLICT OF INTEREST}

The authors certify that they or a member of their immediate family, has no commercial association (i.e., consultancies, stock ownership, equity interest, patent/licensing arrangements, etc.) that might pose a conflict of interest in connection with the submitted manuscript.

\section{REFERENCES}

[1] Nandi S, Kumar M, Saxena M, Saxena AK. The antiviral and antimalarial drug repurposing in quest of chemotherapeutics to combat COVID-19 utilizing structure-based molecular docking. Combinatorial Chemistry \& High Throughput Screening. 2020; 23.

[2] Disease GBD, Injury I, Prevalence C. Global, regional, and national incidence, prevalence, and years lived with disability for 328 diseases and injuries for 195 countries, 1990-2016: a systematic analysis for the Global Burden of Disease Study 2016. The Lancet. 2017; 390: 1211-1259.

[3] Brennan F, Carr D, Cousins M. Access to Pain Management-still very much a Human Right. Pain Medicine. 2016; 17: 1785-1789.

[4] Jukic M, Puljak L. Legal and ethical aspects of pain management. Acta Medica Academica. 2018; 47: 18-26.

[5] Shanthanna H, Cohen SP, Strand N, Lobo C, Eldabe S, Bhatia $\mathrm{A}$, et al. Recommendations on chronic pain practice during the COVID-19 pandemic: a joint statement by American Society of Regional Anesthesia and Pain Medicine (ASRA) and European Society of Regional Anesthesia and Pain Therapy (ESRA). 2020. Available at: https://www.asra.com/page/2903/recommendationson-chronic-pain-practice-during-the-covid-19-pandemic.
[6] Eccleston C, Blyth FM, Dear BF, Fisher EA, Keefe FJ, Lynch ME, et al. Managing patients with chronic pain during the COVID-19 outbreak. Pain. 2020; 161: 889-893.

[7] Shanthanna H, Strand NH, Provenzano DA, Lobo CA, Eldabe S, Bhatia A, et al. Caring for patients with pain during the COVID-19 pandemic: consensus recommendations from an international expert panel. Anaesthesia. 2020; 75: 935-944.

[8] Al-Sadeq DW, Nasrallah GK. The incidence of the novel coronavirus SARS-CoV-2 among asymptomatic patients: a systematic review. International Journal of Infectious Diseases. 2020; 98: 372-380.

[9] Ai T, Yang Z, Hou H, Zhan C, Chen C, Lv W, et al. Correlation of chest CT and RT-PCR testing for coronavirus disease 2019 (COVID-19) in China: a report of 1014 cases. Radiology. 2020; 296: E32-E40.

[10] Nikolai LA, Meyer CG, Kremsner PG, Velavan TP. Asymptomatic SARS coronavirus 2 infection: Invisible yet invincible. International Journal of Infectious Diseases. 2020; 100: 112-116.

[11] Hollander JE, Carr BG. Virtually perfect? Telemedicine for Covid-19. New England Journal of Medicine. 2020; 382: 1679-1681.

[12] Prokop M, van Everdingen W, van Rees Vellinga T, Quarles van Ufford H, Stöger L, Beenen L, et al. CO-RADS: a categorical CT assessment scheme for patients suspected of having COVID-19-definition and evaluation. Radiology. 2020; 296: E97-E104.

[13] Huybens EM, Bus MPA, Massaad RA, Wijers L, van der Voet JA, Delfos NM, et al. What is the preferred screening tool for COVID-19 in asymptomatic patients undergoing a surgical or diagnostic procedure? World Journal of Surgery. 2020; 44: 3199-3206.

[14] Singer JS, Cheng EM, Murad DA, de St. Maurice A, Hines OJ, Uslan DZ, et al. Low prevalence $(0.13 \%)$ of COVID-19 infection in asymptomatic pre-operative/pre-procedure patients at a large, academic medical center informs approaches to perioperative care. Surgery. 2020; 168: 980-986.

[15] Tanacan A, Erol SA, Turgay B, Anuk AT, Secen EI, Yegin GF, et al. The rate of SARS-CoV-2 positivity in asymptomatic pregnant women admitted to hospital for delivery: Experience of a pandemic center in Turkey. European Journal of Obstetrics \& Gynecology and Reproductive Biology. 2020; 253: 31-34.

[16] Mays J, Greninger A, Jerome K, Lynch J, Mathias P. Pre-procedural surveillance testing for SARS-CoV-2 in an asymptomatic population in the seattle region shows low rates of positivity. Journal of Clinical Microbiology. 2020; 58: e01193-20.

[17] Sutton D, Fuchs K, D’Alton M, Goffman D. Universal screening for SARS-CoV-2 in women admitted for delivery. New England Journal of Medicine. 2020; 382: 2163-2164.

[18] Trowbridge S, Wignadasan W, Davenport D, Sarker S, Hunter A, Gidwani $\mathrm{S}$. Is it safe to restart elective day-case surgery? Lessons learned from upper limb ambulatory trauma during the COVID-19 pandemic. Journal of Clinical Orthopaedics and Trauma. 2020; 11: S700-S703.

[19] Cárdenas-Camarena L, Bayter-Marin JE, Durán H, Hoyos A, LópezRomero CO, Robles-Cervantes JA, et al. Elective surgery during SARSCov-2/Covid-19 pandemic. Plastic and Reconstructive Surgery-Global Open. 2020; 8: e2973.

[20] Gammeri E, Cillo GM, Sunthareswaran R, Magro T. Is a "COVID-19free" hospital the answer to resuming elective surgery during the current pandemic? Results from the first available prospective study. Surgery. 2020; 168: 572-577.

[21] Hunter E, Price DA, Murphy E, van der Loeff IS, Baker KF, Lendrem D, et al. First experience of COVID-19 screening of health-care workers in England. the Lancet. 2020; 395: e77-e78.

[22] [COVID-19 with all aspects], Turkish Thoracic Society, COVID19 e-book series, June 2020, ISBN: 978-605-06717-1-1. Available at: https://toraks.org.tr/site/community/downloads/ nDQxie_Cxq8I0otq (Accessed: 8 January 2021).

[23] Kantele A, Lääveri T, Kareinen L, Pakkanen SH, Blomgren K, Mero $\mathrm{S}$, et al. SARS-CoV-2 infections among healthcare workers at Helsinki University Hospital, Finland, spring 2020: Serosurvey, symptoms and risk factors. Travel Medicine and Infectious Disease. 2021; 39: 101949.

[24] Rivett L, Sridhar S, Sparkes D, Routledge M, Jones NK, Forrest S, et al. Screening of healthcare workers for SARS-CoV-2 highlights the role of asymptomatic carriage in COVID-19 transmission. Elife. 2020; 9: e58728. 
[25] COVIDSurg Collaborative. Mortality and pulmonary complications in patients undergoing surgery with perioperative SARS-CoV-2 infection: an international cohort study. The Lancet. 2020; 396: 27-38.

[26] World Health Organization. Global surveillance for COVID-19 caused by human infection with COVID-19 virus: interim guidance. 2020.

[27] Zhang W, Du R, Li B, Zheng X, Yang X, Hu B, et al. Molecular and serological investigation of 2019-nCoV infected patients: implication of multiple shedding routes. Emerging Microbes \& Infections. 2020; 9: 386389.

[28] Rondanelli M, Faliva MA, Miccono A, Naso M, Nichetti M, Riva A, et al. Food pyramid for subjects with chronic pain: foods and dietary constituents as anti-inflammatory and antioxidant agents. Nutrition Research Reviews. 2018; 31: 131-151.

[29] Rautiainen S, Levitan EB, Orsini N, Åkesson A, Morgenstern R, Mittleman MA, et al. Total antioxidant capacity from diet and risk of myocardial infarction: a prospective cohort of women. The American Journal of Medicine. 2012; 125: 974-980.

[30] Memari A, Shariat A, Anastasio AT. Rising incidence of musculoskeletal discomfort in the wake of the COVID-19 crisis. Work. 2020; 66: 751-753.

[31] Shariat A, Anastasio AT, Soheili S, Rostad M. Home-based fundamental approach to alleviate low back pain using myofascial release, stretching, and spinal musculature strengthening during the COVID-19 pandemic. Work. 2020; 67: 11-19.

[32] Dario AB, Moreti Cabral A, Almeida L, Ferreira ML, Refshauge K, Simic M, et al. Effectiveness of telehealth-based interventions in the management of non-specific low back pain: a systematic review with meta-analysis. The Spine Journal. 2017; 17: 1342-1351.

[33] Badalato GM, Kaag M, Lee R, Vora A, Burnett A. Role of telemedicine in urology: contemporary practice patterns and future directions. Urology Practice. 2020; 7: 122-126.

[34] Moo LR, Gately ME, Jafri Z, Shirk SD. Home-based video telemedicine for dementia management. Clinical Gerontologist. 2020; 43: 193-203.

[35] O'Brien KM, Hodder RK, Wiggers J, Williams A, Campbell E, Wolfenden L, et al. Effectiveness of telephone-based interventions for managing osteoarthritis and spinal pain: a systematic review and metaanalysis. PeerJ. 2018; 6: e5846.

[36] Kruse CS, Krowski N, Rodriguez B, Tran L, Vela J, Brooks M. Telehealth and patient satisfaction: a systematic review and narrative analysis. BMJ
Open. 2017; 7: e016242.

[37] Lurie N, Carr BG. The role of telehealth in the medical response to disasters. JAMA Internal Medicine. 2018; 178: 745-746.

[38] Hjelm NM. Benefits and drawbacks of telemedicine. Journal of Telemedicine and Telecare. 2005; 11: 60-70.

[39] Schlumberger HD, Schrinner E. "We have a high research potential". Prof. Dr. med. H. D. Schlumberger, Bayer, and Dr. E. Schrinner, Hoechst, interpret the cooperation of both companies in AIDS research. Interview by T. U. Keil. Fortschritte der Medizin. 1988; 106: 22-23. (In German)

[40] Plein LM, Rittner HL. Opioids and the immune system-friend or foe. British Journal of Pharmacology. 2018; 175: 2717-2725.

[41] Dublin S, Walker RL, Jackson ML, Nelson JC, Weiss NS, Korff M, et al. Use of opioids or benzodiazepines and risk of pneumonia in older adults: a population-based case-control study. Journal of the American Geriatrics Society. 2011; 59: 1899-1907.

[42] Page GG. Immunologic effects of opioids in the presence or absence of pain. Journal of Pain and Symptom Management. 2005; 29: 25-31.

[43] Kim S, Hong S, Yun S, Choi W, Ahn J, Lee YJ, et al. Corticosteroid treatment in critically ill patients with pandemic influenza a/H1N1 2009 infection. American Journal of Respiratory and Critical Care Medicine. 2011; 183: 1207-1214.

[44] Cohen SP, Baber ZB, Buvanendran A, McLean BC, Chen Y, Hooten WM, et al. Pain management best practices from multispecialty organizations during the COVID-19 pandemic and public health crises. Pain Medicine. 2020; 21: 1331-1346.

[45] Liu J, Zheng X, Huang Y, Shan H, Huang J. Successful use of methylprednisolone for treating severe COVID-19. Journal of Allergy and Clinical Immunology. 2020; 146: 325-327.

[46] World Health Organization. Corticosteroids for COVID-19. Available at: https://www. who.int/publications/i/item/WHO-2019-nCoVCorticosteroids-2020.1 (Accessed: 8 January 2021).

How to cite this article: Savas Comlek, Aylin Ordu. Interventional pain management during SARS-CoV-2 pandemic. Signa Vitae. 2021;17(4):178-184. doi:10.22514/sv.2021.008. 